\title{
A Cross-layer Approach for QoS Topology Control in Wireless Ad Hoc Networks
}

\author{
Sk. Mohammad Rokonuzzaman ${ }^{1}$, Ronald Pose $^{1}$ and Iqbal Gondal ${ }^{2}$ \\ Clayton School of IT ${ }^{1}$, Gippsland School of IT $^{2}$ \\ Monash University, VIC 3800, Australia \\ \{Sk.Mohammad.Rokonuzzaman, Ronald.Pose, Iqbal.Gondal\}@infotech.monash.edu.au
}

\begin{abstract}
Wireless ad hoc networks using omni-directional antennas do not scale well due to interference between nearby nodes. Maintaining the QoS of the communications in this type of network is a difficult task. Using multiple narrow beam directional antennas alleviates this problem at the expense of connectivity. Multi-beam Smart Antennas allow the network topology to be adjusted dynamically by adjusting the beamwidth and beam directions to minimize interference and to maximize the number of possible concurrent network communications. This in turn helps to maintain the QoS of the communications. QoS routing has long been used to meet the user requirements by finding appropriate paths to the destinations. We extend this concept to create an Adaptive QoS Topology Control (AQTC) System using Smart Antennas. We use a cross-layer approach to control the topology dynamically where the topology control layer sits between the MAC and the routing protocol. The performance of our protocol has been evaluated using extensive simulations. Simulation results show that different topologies for a set of communications perform differently. AQTC always forms a topology to facilitate the current communications and improves the network throughput and end-to-end delay.
\end{abstract}

Keywords- smart antenna, adaptive QoS topology control, QoS routing protocol.

\section{INTRODUCTION}

Wireless ad hoc networks typically use omni-directional antennas and a common frequency channel. They do not scale well due to interference between nearby nodes. This becomes more adverse in multi-hop networks due to intra-flow and inter-flow interferences [1]. Using collision avoiding CSMA style MAC protocols minimizes such interference, but cannot eliminate it. These protocols try to allow only one of a group of neighboring nodes to transmit at once. In doing so the capacity of the network is limited. It is possible to use a number of different transmission frequencies in a cellular manner to improve the situation and allow nearby nodes to transmit concurrently, but due to the omni-directional transmission pattern network scalability is quite constrained.

Using multiple narrow beam directional antennas alleviates this problem at the expense of the degree of network connectivity. Thus the average path length of communications may increase with highly directional beams, but there is a significant overall gain in network capacity and performance due to its superior scalability.
Quality of service (QoS) routing schemes [2-7] have been employed to make best use of limited network capacity. Typically they involve a search among the possible paths for the subset that meets the QoS requirements of the current communications. In [8] the authors have suggested a preemptive QoS based routing scheme to avoid future QoS violation. But at some point the network may become unable to satisfy all communication requests with their desired QoS even after trying to re-route existing communications to accommodate the new ones.

There has been many works in the literature with directional antennas [9-15]. Directional antennas can reduce the interference problem of wireless communications and have inspired the design of MAC and routing protocols. While using directional antennas, topology control algorithms are needed to select a subset of the neighbors to communicate. Most of the existing topology control algorithms adjust the transmission power to choose these neighbors.

This paper combines the use of multiple beam directional antennas produced by adaptive beamforming smart antennas to adjust the network topology adaptively while maintaining the QoS of the communications. It presents an Adaptive QoS Topology Control (AQTC) algorithm which can change the network topology dynamically by changing the Smart Antenna parameters. By taking into account the QoS requirements of the current communications in the network we can have a new topology which can adapt to the current communication requirements, hence improving the overall network performance. The AQTC algorithm works in the Topology Control layer which sits between the MAC and QoS Routing Protocol. Once the topology is changed, the new paths are verified for the QoS by the routing protocol by sending test data packets.

In the next section we outline our system model. Section III describes the QoS routing protocol in brief. This is followed by the companion AQTC algorithms in Section IV. Section V describes the simulation model used in this study. Then in Section VI we present a summary of simulation results demonstrating the utility of our approach, and a brief discussion of our results. Section VII concludes the paper. 


\section{SYSTEM MODEL}

In our system, each node has one omni-directional beam $(O B)$ and $m$ directional beams $\left(D B_{1}, D B_{2}, \ldots D B_{m}\right)$ like Fig. 1. Each beam has its own transceiver. The beamwidth of the directional beams are $\theta_{1}, \theta_{2}, \ldots \theta_{m}$, the beam directions are denoted by $\alpha_{1}, \alpha_{2}, \ldots \alpha_{m}$ and the transmission ranges are $r_{1}, r_{2}$, ... $r_{m}$.

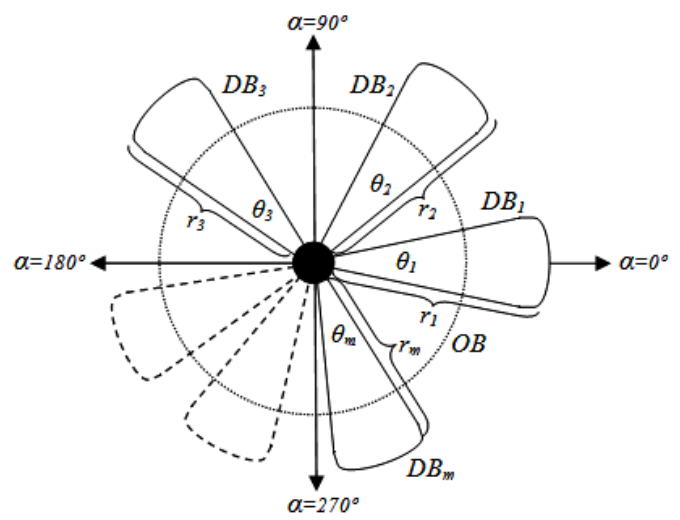

Figure 1. A node with an omni-directional and $\mathrm{m}$ directional beams.

In ad hoc networks, the utilization of the nodes and links are not uniform. So, if one could tailor the network topology to distribute the scarce communication resources to where they are actually needed, one could achieve better performance. To set up a network topology using directional antennas one needs to adjust $\theta$ and $\alpha$ of the $D B$ s of the linked nodes. This would benefit from a priori knowledge of traffic patterns in the network, however this is very difficult to predict. So, the topology control scheme should be able to deal with arbitrary communications. In our network model the network topology is changed dynamically by changing $\theta$ and $\alpha$ of $D B s$ in an adaptive manner to allocate the limited communications resources where they are actually needed, thus providing extra resources for the QoS routing protocol. Modern, electronically steered and beam-forming multi-beam Smart Antennas allow the network topology to be adjusted dynamically in this manner, and maximize the number of possible concurrent communications [16]. But while providing this extra degree of freedom, it may partition the network due to the reallocation of unused beams for the current communications. Thus we need a way to reconfigure the Smart Antennas across the whole network.

This is handled by running the separate omni-directional $(O B)$ control network whose main task is to support the control protocols for adaptive topology control and routing. This control network uses a distinct radio channel which is not used by any directional beams. Its performance is not critical since the more important QoS requirements of user data will be handled by the directional beams forming the topology.

\section{QOS ROUTING}

Routing protocols that provide QoS support in ad hoc networks need to be distributed and should consider the QoS metrics while discovering the route. A modified version of
AODV [17] routing protocol has been used with two QoS metrics, minimum bandwidth required $\left(\mathrm{BW}_{\min }\right)$ and maximum end-to-end delay $\left(\mathrm{D}_{\max }\right)$. It is a reactive routing protocol and each node stores only the next hop in the routing table for the destination nodes.

Each node maintains two routing tables: 1) the Current Routing Table (CRT), and 2) the Alternate Routing Table (ART). The paths discovered in the directional topology are stored in the CRT and used by the current communications and the Re-routing process. These paths are discovered considering the QoS parameters and all the paths in the CRT met the QoS during the route discovery time. On the other hand, the paths discovered in the omni-directional control topology are stored in the ART. These paths are discovered without considering the QoS. The ART is used by the topology control process to look for alternate paths which do not exist in the current directional topology.

During the Route Discovery process each node sends the Route Request (RREQ) message in all the $D B s$ to find paths in the Directional Network. The RREQ packets include the QoS parameters, $\mathrm{BW}_{\min }$ and $\mathrm{D}_{\max }$. Each node keeps a record of the available bandwidths of all the beams. For any link, initial Available Bandwidth, $\mathrm{BW}_{\text {available }}=$ Total Bandwidth of the link and the bandwidth is updated using the following algorithm.

Algorithm 1 Update Available Bandwidth

$\mathrm{BW}_{\text {available }}=\mathrm{BW}_{\text {available }}-\mathrm{BW}_{\text {consumed }}$

Send a message specifying $\mathrm{BW}_{\text {consumed }}$ to the connected nodes of this beam. The connected nodes also update their $\mathrm{BW}_{\text {available }}$ using the above equation.

Algorithm 2 describes the QoS routing process. Here $D_{R R E Q}$ is the end-to-end delay of RREQ packet, $P_{D A T A}$ and $P_{R R E Q}$ are size of data and RREQ packets in bytes respectively, $\delta$ is the time to transmit RTS and CTS messages and $h$ is the number of hops between source and destination. The parameter notToUse includes a list of beam not to be used by the routing process. During the initial routing discovery process this list is normally empty. The paths returned are stored sorted by Shortest Widest Path (SWP). The shortest path is the path with the least number of hops, whereas the widest path is the path with the highest available bandwidth.

\section{Algorithm 2 QoS Routing (Beams notToUse)}

Generate RREQ packets with $\mathrm{BW}_{\text {min }}$ and $\mathrm{D}_{\max }$

The source node sends the RREQ packets on each beam except notToUse, that has $\mathrm{BW}_{\text {available }}>\mathrm{BW}_{\text {min }}$.

Check BW $\mathrm{Bvailable}_{\text {on each beam. }}$

for each beam not in notToUse If $\left(\mathrm{BW}_{\text {available }}>\mathrm{BW}_{\min }\right)$

Call Update Available Bandwidth

Forward the RREQ packet

The source node locks the minimum required bandwidth on

the beam for the duration $2 * \mathrm{D}_{\max }$. end for 
Each intermediate node checks the delay of the RREQ packets so far by checking the timestamp field and also checks the available bandwidth.

if the (delay $\left.<\mathrm{D}_{\max }\right) \& \&\left(\mathrm{BW}_{\text {available }}>\mathrm{BW}_{\text {minimum }}\right)$ send the RREQ packets on these directional links except on the incoming link.

else

don't forward the RREQ packets.

Once the destination is reached, a Route Reply (RREP) packet is sent by the destination towards the source using the reverse path for all RREQ packets received.

if there is no RREP within $2 * \mathrm{D}_{\max }$ release the bandwidth.

else

when a RREP is received lock the bandwidth again for $2^{*} \mathrm{D}_{\max }$.

if there is no data packet from the source within $2 * \mathrm{D}_{\max }$ release the bandwidth.

The RREP packet includes the end-to-end $\left(D_{R R E Q}\right)$ delay of the RREQ packet.

Check all the returned paths for the delay constraint using the formula for data packet delay $\left(D_{D A T A}\right)$,

$$
D_{D A T A}=\left(\frac{P_{D A T A}}{P_{R R E Q}} X D_{R R E Q}\right)+(\delta X h)
$$

Store all the eligible paths in the CRT sorted by SWP.

Then the source node reserves the bandwidth on the appropriate link for the duration of the communication and sends a Bandwidth Reservation (BR) message to the next node and all the intermediate nodes follow the same process. Once the BR packet is sent by the source node, it sends some test data packets to the destination. If these packets are received successfully by the destination node and there is no QoS violation message then the admission control system admits the communication.

The source node also discovers paths for a communication session in the omni-directional control network using Algorithm 3.

Algorithm 3 ART Routing

Send the RREQ packets without specifying QoS parameters in the control network using the omni-directional antenna. Then the basic AODV [14] routing process is followed. Store all the returned paths in ART sorted in increasing order of path length.

\section{ADAPTIVE QOS TOPOLOGY CONTROL}

We have implemented a cross-layer approach for topology control. It is based on the framework as presented in [18]. In this approach we consider QoS Topology Control as a separate protocol layer in the protocol stack between the Routing and
MAC layer. Fig. 2 shows the relationship between the QoS Routing and QoS Topology Control layers.

The network topology for sending data traffic is always formed using the directional beams. The QoS routing protocol uses the current directional topology to find routes which meets the QoS of the communications. But if there is no such route for a new communication even after re-routing some existing communications, then the QoS Routing protocol asks the QoS Topology Control protocol to change the topology to accommodate the new communication if possible. The Topology control layer tries to change the topology and if it is successful then it notifies the routing protocol of the topology change and then the QoS routing protocol uses the new topology to find routes for the new communication. We do not break any existing communications while changing the topology, but try to accommodate the new communication. This can improve the admission ratio.

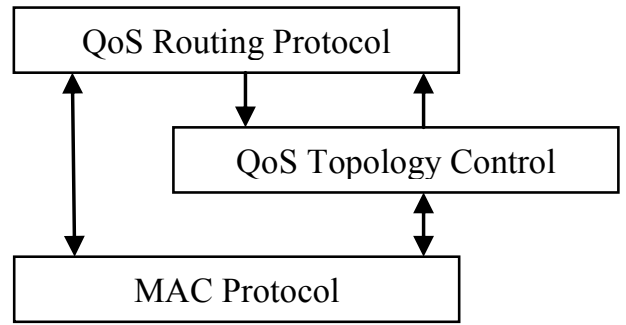

Figure 2. A Cross-layear Approach of Topology Control.

The network topology can be controlled in two ways:

1. Forming an initial fully connected topology during the network initialization phase and then changing the topology dynamically if required for new communications.

2. No initial connected topology is formed, the topology is formed dynamically depending on the communications.

For option 1, during the network initialization phase an initial fully connected topology is formed by the Topology Control Layer so that any initial communications can be handled as they come along. A Local Minimum Spanning (LMST) is build by each node in this case to build a fully connected topology. This topology is initially used by the QoS Routing Protocol to find paths for the initial communications. But, after handling some communications with their required QoS, there could be situations when new communications cannot be admitted as their QoS cannot be met in the current topology with all the current communications running. This depends on the communication pattern of the network and is exacerbated if there are many common beams shared by the current communications.

In this case at first we try to re-route the existing traffic to make room for the new communications with their required QoS. To re-route the existing communications we call the QoS routing process again to find out paths in the current topology that meets the QoS requirements. The paths need to be rediscovered again because the available bandwidth of many 
links may have changed over time and/or there is a change of topology on some portion of the network. The re-routing process is a distributed process and each node updates its CRT and ART locally. The re-routing process returns the best available paths in the current topology. The algorithm is described below.

Algorithm 4 QoS Re-routing (Beams notToUse)

Call QoS Routing Process (notToUse)

Save the returned paths in CRT after the currently used routing entry sorted by SWP.

Stop forwarding packets to MAC layer.

Swap the first two entries in the CRT; the current routing table entry is updated.

Use the first entry as the current route.

Start forwarding packets to MAC layer.

Depending on the communication pattern there may be no routes in the current topology which could meet the required QoS of the new communications. Then the network topology is changed to handle the new communication. The Alternative Routing Table (ART) is used for this purpose. The ART includes all the possible routes of a communication in the Omni-directional topology. These alternate paths and the current direction and beamwidth of the beams are used to change the topology adaptively.

For option 2, we do not form any initial connected topology. Instead we form the topology dynamically as the communications come. So, when a new communication request arrives at the Network Layer, the Routing Protocol looks for a path that meets the required QoS in the current directional topology. If there is no such path, the Network Layer asks the Topology Control Layer to form or modify the current topology for the new communication. The Topology Control Layer uses the ART to find out possible paths and to form the directional topology accordingly.

So, there is a close relationship between the QoS Routing Protocol and the QoS Topology Control Protocol. Once the QoS routing protocol fails to find paths for the new communication with the required QoS even after re-routing the existing traffic, only then the QoS topology control process is initiated. The topology is modified to make room for the new communication as well as for the current communications. Once the new topology is formed the QoS Routing Protocol is initiated by the Topology Control Layer to find paths for the new communication of this topology change.

The aim of the topology control algorithm is to find a suitable topology for the new flow, without breaking any existing flow. Each node has two copies of AQTC, AQTCInitiator and AQTC-Respondent, as described below. AQTCInitiator is used by a node itself, whereas AQTC-Respondent is initiated when a node receives a Request For Beam and Topology (RFBT) message.
Algorithm 5 AQTC-Initiator (source, initiator, session id, destination)

(1) Select the next hop from ART for the destination.

(2) Look for a free $D B_{i}$ or check if the next hop is under the coverage of any $D B_{i}$.

(3) If there is such a $D B_{i}$, lock and direct (if required) the $D B_{i}$ towards the next hop;

Else, check if all the next hops have been tried. If not, go to (1). Otherwise if no free beam has been tried so far, try to free up a $D B_{i}$ using QoS Re-routing process, and go to (1). If no free $D B_{i}$, exit with status Failure.

(4) Send a Request For Beam and Topology (RFBT) message to the next hop and wait for an AFB message.

(5) If there is no ACK For Beam (AFB) message or there is a No Beam Available (NBA) message, go to (1).

(6) Reserve the $D B_{i}$ for the new session and update the CRT with the next hop and wait for a Topology Established (TE) message.

(7) If TE received then forward the TE using directional links until the source node is reached.

(8) If there is no TE message, go to (1).

(9) The source node calls QoS Routing process.

(10) Use a suitable path.

(11) Exit with status Success.

Algorithm 6 AQTC-Respondent (source, sender, session id, destination)

(1) Look for a free $D B_{i}$ or check if the sender is under the coverage of any $D B_{i}$.

(2) If there is such a $D B_{i}$, lock and direct (if required) the $D B_{i}$ towards the sender;

Else, try to free up a $D B_{i}$ using QoS Re-routing process. If no free $D B_{i}$, send a No Beam Available (NBA) message and exit with status Failure.

(3) Send an ACK For Beam (AFB) message to the sender.

(4) Update CRT.

(5) Check if this node is the destination node or has a path in its CRT to the destination.

(6) If so, send a TE message to the sender.

(7) Else call AQTC (source, this node, session id, destination).

As described above, we first re-route the existing traffic to accommodate a new communication and if it fails then we change the topology to accommodate the new communication. If a change of topology is not possible then we do not admit the communication.

\section{Simulatoin MODEL}

The performance of the proposed AQTC algorithm has been evaluated using simulations. We used the GloMoSim [15] simulator which is designed using PARSEC [16]. We established a wireless network of 250 nodes placed randomly on a 4 sq. $\mathrm{km}$ area. We started with 15 communications and then added 15 more communications one by one after each 10 seconds starting at the $10^{\text {th }}$ second. Each communication has 
Constant Bit Rate UDP traffic between randomly selected source-destination pairs. The data packet size is 1024B. We have also tested different packet interarrival times. For QoS, maximum end-to-end delay is 0.05 seconds and minimum bandwidth requirement depends on the packet interarrival time, for e.g. when the packet itnerarrival time is $25 \mathrm{~ms}$ it is $320 \mathrm{kbps}$, for $50 \mathrm{~ms}$ it is $160 \mathrm{kbps}$ and so on. The simulation parameters are listed in Table I.

\begin{tabular}{|c|c|}
\hline Terrain Size & $2000 \mathrm{~m} \times 2000 \mathrm{~m}$ \\
\hline Number of node & 250 \\
\hline Node placement & Random \\
\hline Simulation time & 120 seconds \\
\hline Number of communications & 30 \\
\hline Packet size & 1024B \\
\hline Avg. packet interarrival time & $25 \mathrm{~ms}-150 \mathrm{~ms}$ \\
\hline Traffic type & UDP \\
\hline Interface Queue size & 100 packets \\
\hline Transmission rate & $11 \mathrm{Mbps}$ \\
\hline Propagation-Pathloss model & TWO-RAY \\
\hline MAC protocol & 802.11 \\
\hline Routing protocol & Modified AODV \\
\hline No of directional beams per node & 3 \\
\hline Beamwidth & 30 degree \\
\hline
\end{tabular}

\section{Simulation Results AND Discussion}

In the figures, "Topo_Omni" represents a network with three omni-directional antennas per node each with different radio frequency and "Topo_Dir-(1-6)" mean 6 different fully connected static directional topologies using three $D B s$ per node. AQTC is the proposed algorithm with three $D B s$. For AQTC we do not start with an initial fully connected topology, instead we build the topology dynamically as communication request comes. While simulating different scenarios we found this approach is comparatively better than always starting with a fully connected topology.

Fig. 3 shows the average throughput of all the communications and when the network load is high, AQTC always meets the QoS as it forms a biased topology to meet the QoS of the current communications. The omni-directional topology has the lowest throughput as the delay requirement cannot be met for many packets. For omni-directional transmission nodes need to wait longer to get a share of the channel and eventually packets spend more time on the queues which increases the end-to-end delay. This problem can be reduced if we form a directional topology as can be seen from Fig. 3. All of the 7 directional topologies are performing better than the omni-directional counterpart, but they are not performing in a similar fashion. AQTC and Topo_Dir-3 are always maintaining the QoS, but not all the other directional topologies. This is because AQTC forms or modifies a topology considering the paths from the ART and QoS of the current and new communications so that the communications can find a suitable path. All the other directional topologies, Topo_Dir-(1-6) were just random fully connected topologies and were not formed considering the communications. Topo_Dir-3 is performing like AQTC as the beams in this topology are distributed in a way to balance the load of the current communications like a topology formed by AQTC. For other directional topologies, some links are shared by many communications and also there are overlapping links/beams which force to increase the end-to-end delay.

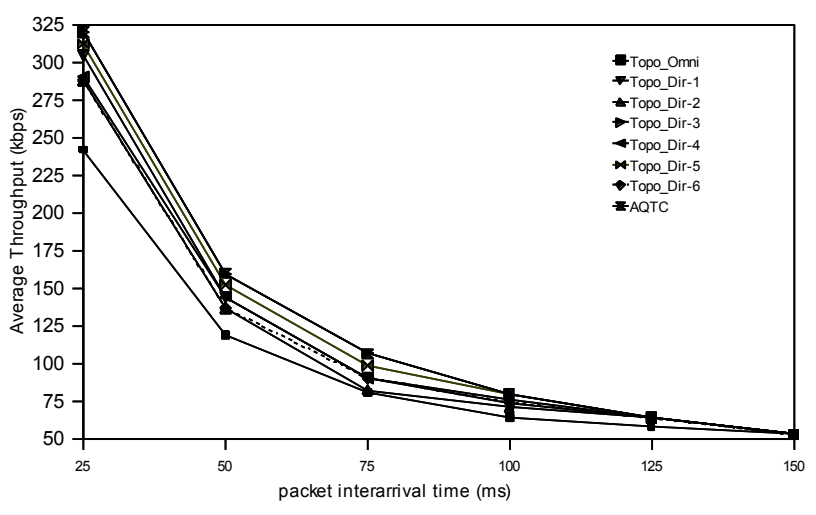

Figure 3. Average throughput vs. packet interval time.

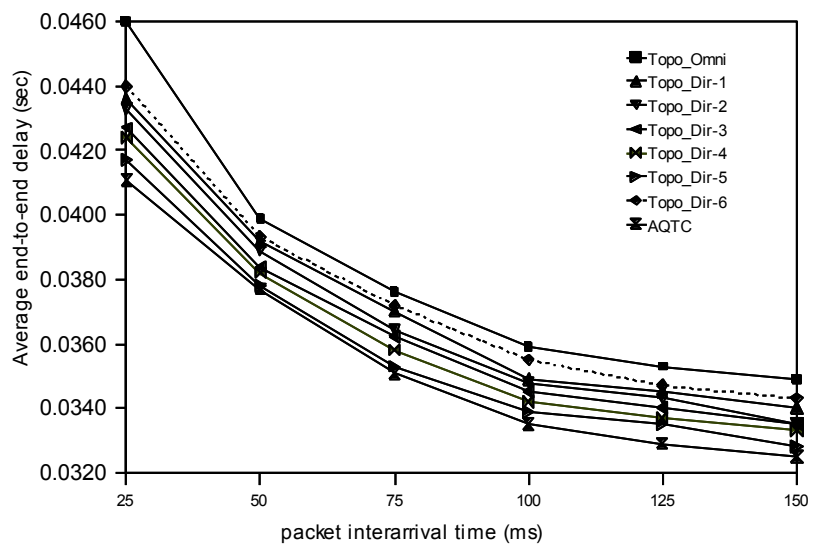

Figure 4. Average end-to-end delay vs. packet interval time.

In Fig. 4 we present the average end-to-end delay of all the communications. The end-to-end delay has been calculated only for successfully received packets which do not cross the QoS delay limit. Though paths are generally longer in a directional topology, all of the directional topologies are incurring less delay than the omni-directional one, but again they are not performing in a similar fashion. While using directional antennas multiple communications are possible in the same neighborhood if a proper topology is formed and packets need to wait less time in the queue. AQTC forms a biased topology considering the shortest path in ART and QoS of the current communications and so better paths can be found which results in least amount of delay.

It is quite evident from Figs. 3 and 4 that, not all the directional topologies that can be formed in a particular ad hoc network perform in a similar fashion. The performance of a topology largely depends on how the topology facilitates the current communications. We have checked the performances of different directional topologies for different communication sets in a network.

In Figs. 5 and 6, we have plotted the throughput for 170 different directional topologies of the network, but for different source-destination combinations. Each small square in these figures represents the throughput of a particular 
network topology. In this case we started with 30 communications with a packet interarrival time of $25 \mathrm{~ms}$. So, the minimum required bandwidth is $320 \mathrm{kbps}$ and at $50^{\text {th }}$ second we formed different directional topologies to compare their performances. These topologies are randomly generated network topologies where at least the source and destination nodes for a particular communication are connected. But not all of them are fully connected topologies.

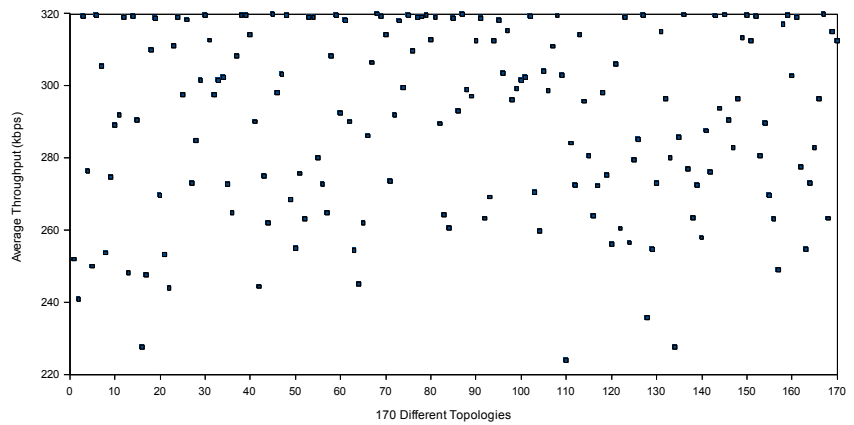

Figure 5. Throughput of 170 different topologies for comm. pattern-1.

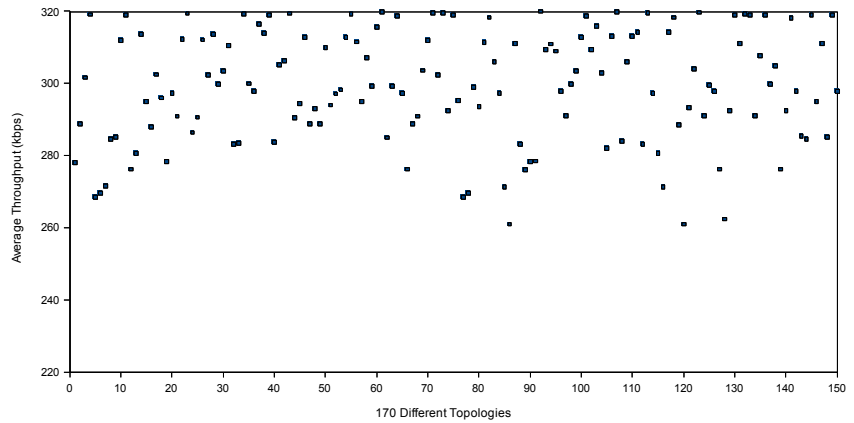

Figure 6. Throughput of 170 different topologies for comm. pattern-2.

In these cases many topologies cannot meet the QoS bandwidth though it is available in the links. This is because many packets cannot meet the QoS delay limit of 0.05 seconds and are not accepted by the destination node. These topologies do not distribute the beams considering the current communications and there are many overlapped beams. Many links are shared by multiple communications due to the unavailability of suitable paths. For these reasons the queuing delay and MAC level delays increase which in turn increase the end-to-end delay. But there are some balanced topologies which can meet the QoS as seen from Fig. 5 and 6 and our AQTC algorithms forms a topology like that as it always considers the suitable paths of the communications from ART to form the topology.

The overhead of the topology control process is due to the RFBT, AFB, NBA and TE control messages which are 20 bytes in size. So the overhead is fairly low. Also these messages are transferred using the omni-directional control channel and do not hamper the transmission of data traffic.

\section{CONCLUSION}

We have presented a cross-layer approach of adaptive topology control with a hop-by-hop routing protocol which increases the network throughput and reduces the end-to-end delay. Future work will focus on other approaches and different aspects of dynamic topology control and evaluating their performance under different network conditions.

\section{REFERENCES}

[1] M. Gong, S. Midkiff, and S. Mao. "A Cross-layer Approach to Channel Assignment in Wireless Ad Hoc Networks," Mobile Networks and Applications, Volume 12, Issue 1, January 2007.

[2] Q. Xue and A. Gunz, "Ad hoc QoS on-demand routing (AQOR) in mobile ad hoc networks," Journal of Parallel and Distributed Computing. Vol. 63, pp. 154-165, 2003.

[3] R. Gupta, Z. Jia, T. Tung, and J. Walrand, "Interference-aware QoS Routing (IQRouting) for Ad-Hoc Networks," in Proc. IEEE GLOBECOM, 2005.

[4] J. Tang, G. Xue and W. Zhang, "Interference-Aware Topology Control and QoS Routing in Multi-Channel Wireless Mesh Networks," in Proc. ACM MobiHoc, 2005.

[5] G. Chauhan, and S. Nandi, "QoS Aware Stable path Routing (QASR) Protocol for MANETs," in Proc. IEEE ICETeT, 2008.

[6] M. M. Islam, R. Pose, and C. Kopp. "Challenges and a Solution to Support QoS for Real-Time Traffic in Multi-Hop Ad-Hoc Networks," in Proc. IEEE and IFIP WoCN, March 2005.

[7] Z. Wang, J. Crowcroft, "Quality of Service Routing for Supporting Multimedia Applications," IEEEJSAC, vol. 14. No 7, September 1996.

[8] S. M. Rokonuzzaman, R. Pose, and I. Gondal, "A Warning Based Preemptive Routing Scheme for QoS Maintenance in Wireless Ad Hoc Networks ", in Proc. ACM PE-WASUN, 2009, Tenerife, Spain.

[9] R. Ramanathan, J. Redi, C. Santivanez, D. Wiggins, and S. Polit, "Ad Hoc Networking with Directional Antennas: A Complete System Solution," IEEE J. Selected Areas in Comm., vol. 23, 2005.

[10] S. Roy, D. Saha, S. Bandyopadhyay, T. Ueda, and S. Tanaka, "A Network-Aware MAC and Routing Protocol for Effective Load Balancing in Ad Hoc Wireless Networks with Directional Antenna," in Proc. ACM MobiHoc, 2003.

[11] R. Choudhury, X. Yang, N. Vaidya, and R. Ramanathan, "Using Directional Antennas for Medium Access Control in Ad Hoc Networks," in Proc. ACM MobiCom, 2002.

[12] Z. Huang and C. Shen, "Multibeam Antenna-Based Topology Control with Directional Power Intensity for Ad Hoc Networks," IEEE Trans. Mobile Computing, vol. 5, no. 5, May 2006.

[13] Z. Zhang, "Pure Directional Transmission and Reception Algorithms in Wireless Ad Hoc Networks with Directional Antennas," in Proc. IEEE ICC, 2005.

[14] S. M. Rokonuzzaman, R. Pose, and I. Gondal, "A Cross-layer Approach for Using Multiple Radio Channels with Directional Beams in a Suburban Ad Hoc Network," in Proc. ATNAC, 2008, Adelaide, Australia, pp 192-197.

[15] S. M. Rokonuzzaman, R. Pose, and I. Gondal, "Multiple Radio Channels and Directional Antennas in Suburban Ad Hoc Networks," in Proc. ISPA, 2008, Sydney, Australia, pp 379-386.

[16] Alexiou, Angeliki, and Haardt, Martin, Smart Antenna Technologies for Future Wireless Systems: Trends and Challenges, IEEE Communication Magazine, September 2004.

[17] Perkins, C., Royer, E. M., and Das, S. Ad hoc On-Demand Distance Vector (AODV) Routing. IETF RFC 3561, 2003.

[18] S. M. Rokonuzzaman, R. Pose, and I. Gondal, "A Framework for a QoS Based Adaptive Topology Control System for Wireless Ad Hoc Networks with Multibeam Smart Antennas", in Proc. AWSN, 2008, Sydney, Australia, pp 940-945.

[19] GloMoSim, http://pcl.cs.ucla.edu/projects/glomosim/ .

[20] PARSEC, http://pcl.cs.ucla.edu/projects/parsec/ . 